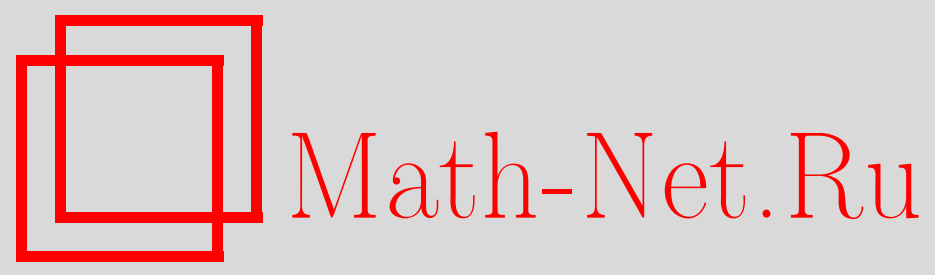

М. М. Хачев, Об одной задаче для уравнения смешанного типа с двумя плоскостями вырождения в бесконечной призматической области, Вестн. Сам. гос. техн. ун-та. Сер. Физ.-мат. науки, 2002, выпуск 16, 15-18

DOI: https://doi.org/10.14498/vsgtu90

Использование Общероссийского математического портала Math-Net.Ru подразумевает, что вы прочитали и согласны с пользовательским соглашением

http: //www. mathnet.ru/rus/agreement

Параметры загрузки:

IP : 107.22 .136 .117

26 апреля 2023 г., 12:32:37 


\section{М.М. Хачев}

\section{ОБ ОДНОЙ ЗАДАЧЕ ДЛЯ УРАВНЕНИЯ СМЕШАННОГО ТИПА С ДВУМЯ ПЛОСКОСТЯМИ ВЫРОЖДЕНИЯ В БЕСКОНЕЧНОЙ ПРИЗМАТИЧЕСКОЙ ОБЛАСТИ}

\section{Доказана теорема об однозначной разрешимости задачи Дирихле для уравнения смешанного типа с} двумя ортогональными плоскостями параболического вырождения.

\section{Постановка задачи и сведение ее к плоской задаче}

Пусть $\Omega$ есть бесконечная призматическая область трехмерного пространства $(x, y, z)$, ограниченная поверхностями

где $\alpha, \beta, l \equiv$ const $>0$.

$$
\begin{gathered}
S_{1}=\{(x, y, z): 0 \leq x \leq l, y=\beta,-\infty<z<+\infty\}, \\
S_{2}=\{(x, y, z): 0 \leq x \leq l, y=-\alpha,-\infty<z<+\infty\}, \\
S_{3}=\{(x, y, z): x=0,-\alpha \leq y \leq \beta,-\infty<z<+\infty\}, \\
S_{4}=\{(x, y, z): x=l,-\alpha \leq y \leq \beta,-\infty<z<+\infty\},
\end{gathered}
$$

В области $\Omega$ рассмотрим уравнение

$$
\operatorname{sgn} y|y|^{m} V_{x x}+\operatorname{sgn} x|x|^{m} V_{y y}+\operatorname{sgn} x y|x y|^{m} V_{z z}=0, \quad m>0,
$$

которое является эллиптическим при $x>0, y>0$ и гиперболическим - при $x>0, y<0$ пространства $(x, y, z)$, за исключением плоскостей $x=0$ и $y=0$, на которых оно параболически вырождается.

Обозначим через $\Omega^{+}=\Omega \cap(y>0), \Omega^{-}=\Omega \cap(y<0)$ эллиптическую и гиперболическую части смешанной области $\Omega$ соответственно.

Задача Дирихле. Найти решение $V \equiv V(x, y, z$,$) уравнения (1) в области \Omega$ со следующими свойствами:

1) $V \in C(\bar{\Omega})$,

2) $V \in C^{1}(\Omega) \cap C^{2}\left(\Omega^{+} \cup \Omega^{-}\right)$за исключением, быть может, плоскостей вырождения

$$
\begin{aligned}
& S_{0}=\{(x, y, z): \quad 0 \leq x \leq l, \quad y=0, \quad-\infty<z<+\infty\}, \\
& S_{3}=\{(x, y, z): \quad x=0, \quad-\alpha \leq y \leq \beta, \quad-\infty<z<+\infty\} ;
\end{aligned}
$$

3) $V$ удовлетворяет краевым условиям:

$$
\begin{aligned}
& \left.V\right|_{S_{1}}=\psi_{1}(x, z), \quad 0 \leq x \leq l, \quad-\infty<z<+\infty ; \\
& \left.V\right|_{S_{2}}=\psi_{2}(x, z), \quad 0 \leq x \leq l, \quad-\infty<z<+\infty ; \\
& \left.V\right|_{S_{3}}=\left.V\right|_{S_{4}}=0, \quad-\alpha \leq y \leq \beta, \quad-\infty<z<+\infty ;
\end{aligned}
$$

$\lim _{z \rightarrow \pm \infty} V=0$ равномерно относительно $(x, y) \in \bar{\Omega} \cap(z=0)$.

Функции $\psi_{1}(x, z)$ и $\psi_{2}(x, z)$ считаем непрерывными в областях своего определения $\left(S_{1}\right.$ и $S_{2}$ соответственно); абсолютно интегрируемыми по $z$ при $-\infty<\mathrm{z}<+\infty, \quad 0 \leq \mathrm{x} \leq 1$; равномерно относительно $x$, стремящимся к нулю при $z \rightarrow \pm \infty$. В дальнейшем, для того чтобы обеспечить принадлежность решения задачи требуемому классу, на $\psi_{1}(x, z)$ и $\psi_{2}(x, z)$ будут наложены еще некоторые ограничения. Принятые же предложения относительно поведения функций $\psi_{1}(x, z), \psi_{2}(x, z)$ и $V(x, y, z)$ позволяют использовать для решения задачи Дирихле метод преобразования Фурье, рекомендованный А.В. Бицадзе [1]. В самом деле, эти ограничения позволяют ввести преобразования Фурье:

$$
V(x, y, z)=\frac{1}{\sqrt{2 \pi}} \int_{-\infty}^{+\infty} u(x, y, \lambda) e^{-i \lambda z} d \lambda
$$


Функция $u(x, y, \lambda)$ выражается через $V(x, y, z)$ с помощью обратного преобразования Фурье:

$$
u(x, y ; \lambda)=\frac{1}{\sqrt{2 \pi}} \int_{-\infty}^{+\infty} V(x, y, z) e^{i \lambda z} d z, \quad \lambda \in R
$$

Введем обозначения: $D=\Omega \cap(z=0), D^{+}=D \cap(y>0), \quad D^{-}=D \cap(y<0)$.

Справедлива следующая лемма:

1) Пусть функция $u(x, y, \lambda)$, являясь решением уравнения

$$
\operatorname{sgn} y|y|^{m} u_{x x}+\operatorname{sgn} x|x|^{m} u_{y y}-\lambda^{2} \operatorname{sgn} x y|x y|^{m} u=0, \quad \lambda \in R,
$$

такова, что интеграл (2) допускает двухкратное дифференцирование по каждому из параметров $x, y, z$. Тогда функция $V(x, y, z)$, определяемая этим интегралом, является решением уравнения (1);

2) если функция $V(x, y, z)$ есть решение уравнения (1), причем такое, что

$$
\lim _{z \rightarrow \pm \infty} V=\lim _{z \rightarrow \pm \infty} V_{z}=0
$$

равномерно относительно $(x, y) \in D$ и интеграл (3) можно дифференцировать два раза по каждому из параметров $x$ и $y$, то функция $u(x, y ; \lambda)$ из (3) удовлетворяет уравнению (4).

Доказательство. Дифференцируя (2), найдем

$$
\begin{aligned}
& 0=\operatorname{sgn} y|y|^{m} V_{x x}+\operatorname{sgn} x|x|^{m} V_{y y}+\operatorname{sgn} x y|x y|^{m} V_{z z} \equiv \\
& \equiv \frac{1}{2 \pi} \int_{-\infty}^{+\infty} e^{i \lambda z}\left[\operatorname{sgn} y|y|^{m} u_{x x}+\operatorname{sgn} x|x|^{m} u_{y y}-\lambda^{2} \operatorname{sgn} x y|x y|^{m} u\right] d \lambda .
\end{aligned}
$$

Отсюда следует справедливость первого утверждения леммы. Далее, из (3) имеем

$$
\begin{aligned}
& 0=\operatorname{sgn} y|y|^{m} u_{x x}+\operatorname{sgn} x|x|^{m} u_{y y}-\lambda \operatorname{sgn} x y|x y|^{m} u \equiv \\
& \equiv \frac{1}{2 \pi} \int_{-\infty}^{+\infty} e^{i \lambda z}\left[\operatorname{sgn} y|y|^{m} V_{x x}+\operatorname{sgn} x|x|^{m} V_{y y}-\lambda^{2} \operatorname{sgn} x y|x y|^{m} V\right] d z .
\end{aligned}
$$

Проинтегрировав последнее слагаемое под знаком интеграла по частям и принимая во внимание, что $\lim _{z \rightarrow \pm \infty} V=\lim _{z \rightarrow \pm}, V_{z}=0$, получим

$$
\frac{1}{2 \pi} \int_{-\infty}^{+\infty}\left[\operatorname{sgn} y|y|^{m} V_{x x}+\operatorname{sgn} x|x|^{m} V_{y y}-\lambda^{2} \operatorname{sgn} x y|x y|^{m} V_{z z}\right] e^{i \lambda z} d z=0
$$

Отсюда следует справедливость второй части леммы.

Задача Дирихле. Найти решение $u \equiv u(x, y ; \lambda)$ уравнения (4) в области $\mathrm{D}$, обладающее следующими свойствами:

1) $u \in C(\bar{D})$;

2) $u \in C^{1}(D) \cap C^{2}\left(D^{+} \cup D^{-}\right)$за исключением, быть может, характеристик уравнения (4) ;

3)удовлетворяет краевым условиям:

$$
\begin{aligned}
& u(x, \beta ; \lambda)=\psi_{1}(x, \lambda), \quad u(x,-\alpha ; \lambda)=\psi_{2}(x, \lambda), \quad 0 \leq x \leq l, \quad \lambda \in R, \\
& u(0, y ; \lambda)=0, \quad u(l, y ; \lambda)=0, \quad-\alpha \leq y \leq \beta, \quad \lambda \in R,
\end{aligned}
$$

где

$$
\psi_{j}(x, \lambda)=\frac{1}{\sqrt{2 \pi}} \int_{-\infty}^{+\infty} \Psi_{j}(x, z) e^{i \lambda z} d z, \quad j=1,2 ; \quad \lambda \in R
$$

Таким образом, сопоставляя задачи Дирихле в областях $\Omega$ и $D$, видим, что доказательство существования решения задачи Дирихле в области для уравнения (1) сводится к доказательству разрешимости задачи Дирихле в области $D$ для уравнения (4) при любом действительном значении параметра $\lambda \in R$.

Относительно граничных функций $\psi_{1}(x, \lambda)$ и $\psi_{2}(x, \lambda)$ предполагаем, что непериодические функции $\bar{\psi}_{1}(x, \lambda)$ и $\bar{\psi}_{2}(x, \lambda)$ при фиксированных значениях $\lambda \in R$, полученные от $\psi_{1}(x, \lambda)$ и $\psi_{2}(x, \lambda)$ путем нечетного продолжения на отрезок $[-l, 0]$, а за тем на всю ось с периодом $2 l$, 
являются трижды непрерывно дифференцируемыми на $R$ при любом действительном значении параметра $\lambda$.

Обозначим через

$$
a_{m}=\frac{2}{l^{2} J_{v+1}^{2}\left(j_{m, v}\right)} \int_{0}^{l} t \cdot f(t) J_{v}\left(j_{m, v} t\right) d t
$$

где $v+\frac{1}{2} \geq 0, j_{m, v}$ - корни функции Бесселя первого рода порядка $v$.

Справедлива следующая теорема.

Т е о р е м а Гобсона [2]. Пусть:

1) $f(t)$ произвольная функция, заданная на интервале $(0, l)$;

2) $\int_{0}^{l} \sqrt{t} f(t) d t$ существует и абсолютно сходится;

3) $\mathrm{x}$ - какая-нибудь внутренняя точка интервала $(a, b)$, такого, что $0<\mathrm{a}<\mathrm{b}<1$ и $f(t)$ имеет на нем ограниченное полное изменение.

Тогда ряд Фурье- Бесселя $\sum_{m=1}^{\infty} a_{m} J_{v}\left(j_{m, v} x\right)$

сходится и сумма его равна $\frac{1}{2}[\mathrm{f}(\mathrm{x}-0)+\mathrm{f}(\mathrm{x}+0)]$. Если $f(0)=f(l)=0$, то сходимость имеет место в $[0, l]$.

Введем функцию:

$$
E_{n}\left(\alpha_{1}, \beta_{1}\right) \equiv \sqrt{\alpha \beta}\left[J_{p}\left(\alpha_{1} \mu_{n}(\lambda)\right) I_{-p}\left(\beta_{1} \mu_{n}(\lambda)\right)+J_{-p}\left(\alpha_{1} \mu_{n}(\lambda)\right) I_{p}\left(\beta_{1} \mu_{n}(\lambda)\right)\right],
$$

где $\quad \alpha_{1}=2 p \sqrt[2 p]{\alpha}, \beta_{1}=2 p \sqrt[2 p]{\beta}, \mu_{n}^{2}(\lambda)=\lambda^{2}+k_{n}^{2}, \lambda \in R, k_{n}=j_{p, n} /(2 p \sqrt[2 p]{l}), j_{p, n}$-корни $\quad$ функции Бесселя первого рода порядка $p=\frac{1}{2+m}$.

Имеет место следующая основная теорема.

T е о р е м а. Пусть:

1) постоянные величины $\alpha, \beta, l$ таковы, что для всех $n \in N$ и фиксированных значений $\lambda \in R$

$$
E_{n}\left(\alpha_{1}, \beta_{1}\right) \neq 0
$$

2) функции $\psi_{1}(x, \lambda)$ и $\psi_{2}(x, \lambda)$ удовлетворяют условиям теоремы Гобсона при любом действительном значении параметра $\lambda \in R$;

3 ) для всех $n=1,2,3 \ldots$ и фиксированных значений $\lambda \in R$ имеет место неравенство

$$
\inf _{n} \sqrt{\mu_{n}(\lambda)}\left|E_{n}\left(\alpha_{1}, \beta_{1}\right)\right|>0 .
$$

Тогда существует единственное решение задачи Дирихле для уравнения (4) в области D, представимое в виде

где

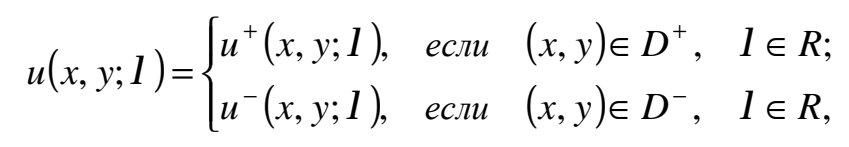

$$
\begin{gathered}
u^{+}(x, y ; \lambda)=\sum_{n=1}^{\infty}\left[\frac{M_{n}\left(\alpha_{1}, y\right)}{E_{n}\left(\alpha_{1}, \beta_{1}\right)} \psi_{1 n}(\lambda)+\frac{N_{n}\left(\beta_{1}, y\right)}{E_{n}\left(\alpha_{1}, \beta_{1}\right)} \psi_{2 n}(\lambda)\right]\left(\sqrt{x} J_{p}\left(j_{p, n} \sqrt[2 p]{\frac{x}{l}}\right),\right. \\
u^{-}(x, y ; \lambda)=\sum_{n=1}^{\infty}\left[\frac{K_{n}\left(\alpha_{1}, y\right)}{E_{n}\left(\alpha_{1}, \beta_{1}\right)} \psi_{1 n}(\lambda)+\frac{Z_{n}\left(\beta_{1}, y\right)}{E_{n}\left(\alpha_{1}, \beta_{1}\right)} \psi_{2 n}(\lambda)\right] \sqrt{x} J_{p}\left(j_{p, n} \sqrt[2 p]{\frac{x}{l}}\right), \\
\mathrm{M}_{\mathrm{n}}\left(\alpha_{1}, \mathrm{y}\right)=\sqrt{\alpha \mathrm{y}}\left[\mathrm{I}_{\mathrm{p}}(\overline{\mathrm{y}}) \mathrm{J}_{-\mathrm{p}}(\bar{\alpha})+\mathrm{I}_{-\mathrm{p}}(\overline{\mathrm{y}}) \mathrm{J}_{\mathrm{p}}(\bar{\alpha})\right], \mathrm{N}_{\mathrm{n}}\left(\beta_{1}, \mathrm{y}\right)=\sqrt{\beta \mathrm{y}}\left[\mathrm{I}_{\mathrm{p}}(\bar{\beta}) \mathrm{I}_{-\mathrm{p}}(\overline{\mathrm{y}})-\mathrm{I}_{\mathrm{p}}(\overline{\mathrm{y}}) \mathrm{I}_{-\mathrm{p}}(\bar{\beta})\right], \\
\mathrm{K}_{\mathrm{n}}\left(\alpha_{1}, \mathrm{y}\right)=\sqrt{-\alpha \mathrm{y}}\left[\mathrm{J}_{\mathrm{p}}(\bar{\alpha}) \mathrm{J}_{-\mathrm{p}}(\overline{\mathrm{y}})-\mathrm{J}_{-\mathrm{p}}(\bar{\alpha}) \mathrm{J}_{\mathrm{p}}(\overline{\mathrm{y}})\right], \mathrm{Z}_{\mathrm{n}}\left(\beta_{1}, \mathrm{y}\right)=\sqrt{-\beta \mathrm{y}}\left[\mathrm{J}_{\mathrm{p}}(\overline{\mathrm{y}}) \mathrm{I}_{-\mathrm{p}}(\bar{\beta})-\mathrm{J}_{-\mathrm{p}}(\overline{\mathrm{y}}) \mathrm{I}_{\mathrm{p}}(\bar{\beta})\right], \\
\bar{y}=2 p \sqrt[2 p]{|y|} \mu_{n}(\lambda), \quad \bar{\alpha}=\alpha_{1} \mu_{n}(\lambda), \bar{\beta}=\beta_{1} \mu_{n}(\lambda) .
\end{gathered}
$$

Теорема доказывается методом разделения переменных с учетом свойств бесселевых функций [2]. 


\section{Единственность и существование решения пространственной задачи Дирихле}

Определение 1. Решением задачи Дирихле в области $\Omega$ для уравнения (1) класса $F$ будем называть функцию $\mathrm{V}(\mathrm{x}, \mathrm{y}, \mathrm{z})$, определяемую формулой $(2)$, в которой $u(x, y ; \lambda)$ является решением задачи Дирихле в области D для уравнения (4) класса $C(\bar{D}) \cap C^{1}(D) \cap C^{2}\left(D^{+} \cup D^{-}\right)$.

T e o p e м а 1. В классе $F$ существует не более одного решения задачи Дирихле для уравнения (1) в области $\Omega$, если $\left.V\right|_{\partial \Omega}=0$, где $\partial \Omega-$ граница области $\Omega$.

Доказательство. Пусть $\psi_{1}(x, z) \equiv 0, \psi_{2}(x, z) \equiv 0$. Тогда из формул

$$
\psi_{j}(x, \lambda)=\frac{1}{\sqrt{2 \pi}} \int_{-\infty}^{+\infty} \Psi_{j}(x, z) e^{i \lambda z} d z \quad(j=1,2)
$$

следует, что $\psi_{j}(x, \lambda) \equiv 0, \quad j=1,2, \lambda \in R$. Следовательно, $u(x, y ; \lambda) \equiv 0$ в $D$ и из вышеприведенной формулы получаем, что $V \equiv 0$ в $\Omega$. Теорема доказана.

Определение 2. Функции $\psi_{1}(x, z), \quad \psi_{2}(x, z)$ принадлежат классу $Q_{\lambda}$, если:

$1)$ они непрерывны в области своего определения $\left(S_{1}\right.$ и $S_{2}$ соответственно); абсолютно интегрируемы по $z$ при $-\infty<z<+\infty, 0 \leq x \leq l$; равномерно относительно $x$ стремятся к нулю при $z \rightarrow \pm \infty$;

2) их преобразования Фурье

$$
\Psi_{j}(x, \lambda)=\frac{1}{\sqrt{2 \pi}} \int_{-\infty}^{+\infty} \Psi_{j}(x, z) e^{i \lambda z} d z, \quad j=1,2
$$

при $|\lambda| \rightarrow+\infty$ имеют оценки

$$
\psi_{1}(x, \lambda)=O\left(\frac{1}{|\lambda|^{1+\varepsilon} e^{|\lambda|}}\right), \quad \Psi_{2}(x, \lambda)=O\left(\frac{1}{|\lambda|^{1+\varepsilon} e^{|\lambda|}}\right), \quad \varepsilon>0 .
$$

Если функции $\Psi_{j}(x, z) \in Q_{\lambda}, \quad j=1,2$, то из формул (6) и (7) с учетом асимптотических представлений бесселевых функций

$$
I_{ \pm v}(z)=\frac{e^{z}}{\sqrt{2 \pi z}}, \quad J_{ \pm v}(z)=\sqrt{\frac{2}{\pi z}} \cos \left(z \mathrm{~m}_{\frac{\pi v}{2}}-\frac{\pi}{4}\right)
$$

и оценок (8) легко получаем оценку для функций $u(x, y ; \lambda)$ при $|\lambda| \rightarrow+\infty$ :

$$
u(x, y ; \lambda)=O\left(\frac{1}{|\lambda|^{1+\varepsilon}}\right), \quad(x, y) \in \bar{D},
$$

которая обеспечивает существование интеграла (2).

T е о р е м а 2. Если $\Psi_{\mathrm{j}}(\mathrm{x}, \mathrm{z}) \in \mathrm{Q}_{\lambda}(\mathrm{j}=1,2)$, то существует единственное решение класса $F$ задачи Дирихле в области $\Omega$ для уравнения (1).

Доказательство теоремы 2 следует из теоремы 1, леммы и формулы (5).

\section{БИБЛИОГРАФИЧЕСКИЙ СПИСОК}

1. Бицадзе А.В. Об одном трехмерном аналоге задачи Трикоми // Сибир. мат. журн. 1962. Т.3. Вып. 5.С. 642-644.

2. Ватсон Г.Н. Теория бесселевых функций. Ч.1. М.: ИЛ, 1949. 480 с. 